\title{
Computable Analysis of Linear Rearrangement Optimization ${ }^{\star}$
}

\author{
Amin Farjudian 1[0000-0002-1879-0763] \\ School of Computer Science \\ University of Nottingham Ningbo China \\ Amin. Farjudian@nottingham. edu.cn \\ www.nottingham.edu.cn/en/science-engineering/staffprofile/amin-farjudian.aspx
}

\begin{abstract}
Optimization problems over rearrangement classes arise in various areas such as mathematics, fluid mechanics, biology, and finance. When the generator of the rearrangement class is two-valued, they reduce to shape optimization and free boundary problems which can exhibit intriguing symmetry breaking phenomena. A robust framework is required for computable analysis of these problems. In this paper, as a first step towards such a robust framework, we provide oracle Turing machines that compute the distribution function, decreasing rearrangement, and linear rearrangement optimizers, with respect to functions that are continuous and have no significant flat zones. This assumption on the reference function is necessary, as otherwise, the aforementioned operations may not be computable. We prove that the results can be computed to within any degree of accuracy, conforming to the framework of Type-II Theory of Effectivity.
\end{abstract}

Keywords: Computable Analysis · Rearrangements of functions · Optimization.

\section{Introduction}

The aim of the current paper is to lay the foundation for computability and complexity analysis of optimization problems over rearrangement classes, in the framework of Type-II Theory of Effectivity (TTE) [18].

The theory of rearrangements of functions may be traced back to 1899 , when it was introduced as a framework for the study of a problem in hydrostatics [17]. In the following decades, even though it attracted attention from some of the most prominent mathematicians of the twentieth century, it remained a peripheral tool in mathematical analysis, until it re-emerged in the 1970s in the work of Benjamin [1]. Specifically, a problem in fluid mechanics related to steady vortices was formulated by Benjamin as an optimization problem over a rearrangement class.

In response, G. R. Burton laid out a theory for optimization of convex functionals over rearrangement classes [3]5]. Although PDE-constrained rearrangement optimization problems have their origins in fluid mechanics, the abstract formulation of these problems has shown greater potential, and Burton's theory has been used in the

* This is a preprint of the paper [13]. The final authenticated version is available online at https://doi.org/10.1007/978-3-030-14812-6_11 
study of PDE-constrained rearrangement optimization problems in several areas, e. g., finance [12], free boundary problems [8], non-local problems [11], population biology [10], and eigenvalue problems [9], to name a few. Of special interest is the case when the generator of the rearrangement class is a two-valued function. Rearrangement optimization problems with two-valued generators form some important examples of shape optimization and free boundary problems.

In virtually all but exceptional cases, analytic solutions do not exist for these problems. Even when existence of (not necessarily analytic) solutions is guaranteed by the theory, there are no rigorous computational frameworks for computability and complexity analysis of the solutions. Apart from rare cases, even qualitative accounts of the optimal shapes are missing from the literature. In summary, assuming the existence of a solution is guaranteed, in the majority of cases, the answers to the following questions are still unknown:

(1) Is the optimal solution computable?

(2) Does there exist a Type-II Turing machine, which takes the input parameters of the problem, and returns the optimal solution? Note that this is the uniform version of Question (1).

(3) What are the robustness properties of the optimal solutions?

The following question arises in the related shape optimization problems:

(4) If symmetry breaking occurs, how does it occur? If symmetry is preserved for the $n$-dimensional ball $B$, but breaks for the given reference domain $\Omega$, and if we obtain $\Omega$ from $B$ through a smooth deformation, at what point does symmetry break?

\subsection{Contributions of the paper}

On the computational side of PDE-constrained rearrangement optimization problems, only sporadic attempts have been made, with the main focus on numerical algorithms based on floating-point arithmetic [7|14[11[10]. The current paper is meant to serve as a starting point towards answering questions of the type just listed. We aim to lay the foundation for a computational framework that allows us to study rearrangement optimization problems in a validated setting.

Specifically, we provide oracle Turing machines that compute the distribution function, decreasing rearrangement, and linear rearrangement optimizers, with respect to functions that are continuous and have no significant flat zones. This assumption on the reference function is necessary, as otherwise, the aforementioned operations may not be computable. Furthermore, the reference functions are solutions of the constraint partial differential equations (PDEs), which, in virtually all applications, satisfy the assumption on continuity and flat zones. For this reason, many of the results in the literature on rearrangement optimization problems-including Burton's seminal papers [3]5] are formulated with respect to this assumption.

We prove that the results can be computed to within any degree of accuracy, conforming to the framework of Type-II Theory of Effectivity.

Remark 1. It should be noted that the existence of linear rearrangement optimizers is a fundamental result in the theory of rearrangements of functions (Lemma 1 (iv)). Our aim here is to investigate its computability. 


\subsection{Our approach}

Throughout the paper, we make sure that the arguments adhere to Type-II Theory of Effectivity, as presented in [18]. Yet, instead of using the concepts and notations of [18], we work directly with the discretizations of the domains and maps involved. We hope that this makes the content accessible to a broader audience, as we believe that this direct approach allows us to express our algorithms in a way that is more intuitive.

\subsection{Structure of the paper}

- In Sect. 2, we present the background concepts and results from the theory of rearrangements, together with some basic notations that we will be adopting for the remainder of the paper.

- Sect. 3 contains our main results regarding computable analysis of the distribution function and the decreasing rearrangements.

- In Sect. 4 we present our main results regarding linear rearrangement optimization.

- Sect. 5 discusses some generalizations of the main results to other domains and dimensions.

- We conclude the paper in Sect. 6. where we also discuss some future work on computable analysis of rearrangement optimization problems.

\section{Preliminaries}

The material in this section includes some basic concepts and results from the theory of rearrangements of functions. We will also establish some notations that we will be adopting throughout the paper.

\subsection{Rearrangement theory}

Let $(\Omega, \Sigma, \mu)$ be a measure space, in which $\Omega$ is a non-empty set, $\Sigma$ is a $\sigma$-algebra on $\Omega$, and $\mu$ is a positive measure on $\Omega$ satisfying $\mu(\Omega)<\infty$.

Definition 1 (distribution function $\lambda_{f}$ ). For a real measurable $f: \Omega \rightarrow \mathbb{R}$, the distribution function $\lambda_{f}: \mathbb{R} \rightarrow \mathbb{R}$ is defined by: $\forall s \in \mathbb{R}: \lambda_{f}(s):=\mu\left(f^{-1}[s, \infty)\right)$.

Definition 2 (rearrangement class $\left.\mathcal{R}_{\boldsymbol{\Omega}}\left(\boldsymbol{f}_{\mathbf{0}}\right)\right)$. Let $\left(\Omega_{0}, \Sigma_{0}, \mu_{0}\right)$ and $(\Omega, \Sigma, \mu)$ be two measure spaces, such that $\mu_{0}\left(\Omega_{0}\right)=\mu(\Omega)$.

(a) We say that $f_{0}: \Omega_{0} \rightarrow \mathbb{R}$ and $f: \Omega \rightarrow \mathbb{R}$ are rearrangements of each other if and only if $\forall s \in \mathbb{R}: \mu_{0}\left(f_{0}^{-1}[s, \infty)\right)=\mu\left(f^{-1}[s, \infty)\right)$.

(b) The rearrangement class $\mathcal{R}_{\Omega}\left(f_{0}\right)$ generated by $f_{0}$ is defined as follows:

$$
\mathcal{R}_{\Omega}\left(f_{0}\right):=\left\{f: \Omega \rightarrow \mathbb{R} \mid f \text { is a rearrangement of } f_{0}\right\} .
$$

Whenever $\left(\Omega_{0}, \Sigma_{0}, \mu_{0}\right)=(\Omega, \Sigma, \mu)$, we may simply write $\mathcal{R}\left(f_{0}\right)$. If both $f_{0}$ and $\Omega$ are clear from the context, we may just use the symbol $\mathcal{R}$ to denote the rearrangement class. 
For any Lebesgue-measurable $\Omega \subseteq \mathbb{R}^{n}$ and $f: \Omega \rightarrow \mathbb{R}$, we let $\|f\|_{p}$ denote the usual $L^{p}$ norm:

$$
\|f\|_{p}:= \begin{cases}\left(\int_{\Omega}|f(x)|^{p} \mathrm{~d} x\right)^{\frac{1}{p}}, & \text { if } p \in[1, \infty), \\ \operatorname{ess} \sup \{|f(x)| \mid x \in \Omega\}, & \text { if } p=\infty .\end{cases}
$$

For every $p \geq 1$, we let $q$ denote its conjugate exponent satisfying $1 / p+1 / q=1$ when $p>1$, and $q=\infty$ when $p=1$.

Henceforth, we make the following assumptions:

- $\Omega$ denotes a bounded, open, and connected domain in $\mathbb{R}^{n}$;

- $\Sigma$ denotes the Lebesgue $\sigma$-algebra over $\Omega$, with $\mu$ denoting the Lebesgue measure. Indeed, for simplicity, we denote the $n$-dimensional Lebesgue measure of any Lebesgue-measurable $E \subseteq \mathbb{R}^{n}$ by $|E|$.

- $\Omega_{0}$ denotes the open interval $(0,|\Omega|)$.

Definition $3\left(f^{\Delta}, f_{\Delta}\right.$ : non-increasing and non-decreasing rearrangements). For $a$ real measurable $f: \Omega \rightarrow \mathbb{R}$ :

(i) The (essentially unique) non-increasing rearrangement $f^{\Delta}$ of $f$ is defined on $\Omega_{0}$ by $f^{\Delta}(s):=\sup \left\{\alpha \in \mathbb{R} \mid \lambda_{f}(\alpha) \geq s\right\}$. In case $f$ can be extended to $\bar{\Omega}$, with an essential infimum $a$ and an essential supremum $b$, we extend $f^{\Delta}$ to $\overline{\Omega_{0}}$ by letting $f^{\Delta}(0):=b$ and $f^{\Delta}(|\Omega|):=a$.

(ii) The (essentially unique) non-decreasing rearrangement $f_{\Delta}$ of $f$ is defined on $\Omega_{0}$ by $f_{\Delta}(s):=f^{\Delta}(|\Omega|-s)$.

Definition 4 (significant flat zones). A measurable function $f: \Omega \rightarrow \mathbb{R}$ is said to have no significant flat zones on $\Omega$ if $\forall c \in \mathbb{R}:\left|f^{-1}(c)\right|=0$.

The following is very easy to establish:

Proposition 1. (i) If $f$ is continuous, then $\lambda_{f}$ has no significant flat zones.

(ii) If $f$ has no significant flat zones, then $f^{\Delta}$ is decreasing, and $f_{\Delta}$ is increasing.

(iii) If $f$ is continuous and has no significant flat zones, then $f^{\Delta}$ and $\lambda_{f}$ are both continuous, decreasing, and are the inverses of each other.

We will consider linear rearrangement optimization against functions that have no significant flat zones. This condition guarantees uniqueness of solutions, and provides a convenient optimality condition, as summarized in the following lemma:

Lemma 1. Assume that $f_{0} \in L^{p}\left(\Omega_{0}\right)$, and let $\mathcal{R}:=\mathcal{R}_{\Omega}\left(f_{0}\right)$ be its rearrangement class over $\Omega$. Then:

(i) $\mathcal{R} \subseteq L^{p}(\Omega)$.

(ii) $\forall f \in \mathcal{R}:\|f\|_{p}=\left\|f_{0}\right\|_{p}$.

(iii) The weak closure of $\mathcal{R}$ in $L^{p}(\Omega)$, denoted by $\overline{\mathcal{R}}$, is weakly compact and convex.

(iv) For every $h \in L^{q}(\Omega)$, the linear functional $L_{h}: L^{p}(\Omega) \rightarrow \mathbb{R}$ defined by:

$$
L_{h}(f):=\int_{\Omega} f(x) h(x) \mathrm{d} x
$$

has a maximizer $\hat{f}$ over $\mathcal{R}$. 
(v) If $\hat{f}$ is the unique maximizer of the linear functional $L_{h}$ over $\mathcal{R}$, then it is the unique maximizer of $L_{h}$ over all of $\overline{\mathcal{R}}$. Moreover, $\hat{f}=\hat{\psi}(h)$, almost everywhere in $\Omega$, for some non-decreasing function $\hat{\psi}$.

(vi) For any $h \in L^{q}(\Omega)$ with no significant flat zones, there exists a non-decreasing function $\hat{\psi}$ such that $\hat{\psi}(h) \in \mathcal{R}$, and $\hat{f}:=\hat{\psi}(h)$ is the unique maximizer of the linear functional $L_{h}$ defined in $(1)$ over $\overline{\mathcal{R}}$. Furthermore:

$$
\hat{\psi}=f_{0}^{\Delta} \circ \lambda_{h} .
$$

(vii) Items (iv), (v), and (vi) remain valid if one replaces 'maximizer' with 'minimizer', and 'non-decreasing function $\hat{\psi}$ ' with 'non-increasing function $\breve{\psi}$ ', in which case, equation 27 becomes $\breve{\psi}=f_{04} \circ \lambda_{h}$.

Proof. See [3] and [4].

We will also refer to the following results related to non-increasing rearrangements from the literature:

Lemma 2. Assume that $1 \leq p<\infty$. Then:

(i) For any given $f \in L^{p}(\Omega)$, there exists a measure-preserving map $\rho: \Omega \rightarrow[0,|\Omega|]$ such that $f=f^{\Delta} \circ \rho$.

(ii) $\forall f, g \in L^{p}(\Omega):\left\|f^{A}-g^{A}\right\|_{p} \leq\|f-g\|_{p}$.

Proof. (i) See [4, Lemma 2.4].

(ii) See [6], or [4] Lemma 2.7].

\subsection{Further definitions and notations}

The set of dyadic numbers will be denoted by $\mathbb{D}$, i. e., $\mathbb{D}:=\left\{p / 2^{n} \mid p \in \mathbb{Z}, n \in \mathbb{N}\right\}$. For any $n, k \in \mathbb{N}$, let $M_{k}^{n}$ be the meshgrid with granularity $k$ over $[0,1]^{n}$ whose vertices are:

$$
\left\{\left(\frac{p_{1}}{2^{k}}, \frac{p_{2}}{2^{k}}, \ldots, \frac{p_{n}}{2^{k}}\right) \mid p_{1}, p_{2}, \ldots, p_{n} \in\left\{0,1, \ldots, 2^{k}\right\}\right\} .
$$

By an element (or a cell) in a meshgrid $M_{k}^{n}$, we mean a compact box of the form:

$\left[\frac{p_{1}}{2^{k}}, \frac{p_{1}+1}{2^{k}}\right] \times\left[\frac{p_{2}}{2^{k}}, \frac{p_{2}+1}{2^{k}}\right] \times \cdots \times\left[\frac{p_{n}}{2^{k}}, \frac{p_{n}+1}{2^{k}}\right], \quad p_{1}, p_{2}, \ldots, p_{n} \in\left\{0,1, \ldots, 2^{k}-1\right\}$.

Clearly, every meshgrid $M_{k}^{n}$ is the union of $2^{k n}$ such cells, and for each such box $S$ :

$$
\forall x, y \in S: \quad\|x-y\|_{\infty} \leq 2^{-k},
$$

in which $\|\cdot\|_{\infty}$ is the sup norm on $\mathbb{R}^{n}$. When $n$ is clear from the context, we may just write $M_{k}$.

A box $\prod_{i=1}^{n}\left[a_{i}, b_{i}\right]$ with rational vertices will be represented by the following element of $\mathbb{Q}^{2 n}$ :

$$
\left(a_{1}, b_{1}, a_{2}, b_{2}, \ldots, a_{n}, b_{n}\right) .
$$

For any set $T$, we denote the set of finite subsets of $T$ as $\mathcal{P}_{\text {fin }}(T)$. For instance, a finite set of two-dimensional rational boxes is an element of $\mathcal{P}_{\text {fin }}\left(\mathbb{Q}^{4}\right)$. 
Definition 5 (simple step function). Let $q>0$ be a rational number. A function $f:[0, q] \rightarrow \mathbb{R}$ is said to be a simple step function if for some $n \in \mathbb{N}$, there is a set $\left\{x_{0}, x_{1}, \ldots, x_{n}, y_{1}, \ldots, y_{n}\right\} \subseteq \mathbb{Q}$ such that:

(a) $0=x_{0}<x_{1}<\cdots<x_{n}=q$.

(b) $f(0)=y_{1}$ and $\forall x \in\left(x_{i-1}, x_{i}\right]: f(x)=y_{i}$ for $1 \leq i \leq n$.

We denote the closure and interior of a set $A$ by $\bar{A}$ and $A^{\circ}$, respectively. The characteristic function of a subset $A$ of a reference set $Y$ will be denoted as $\chi_{A}$, which is defined as:

$$
\forall y \in Y: \quad \chi_{A}(y):= \begin{cases}0, & \text { if } y \notin A, \\ 1, & \text { if } y \in A .\end{cases}
$$

Another concept that we will refer to quite frequently is that of a modulus of continuity of a function:

Definition 6 (modulus of continuity). Let $\left(X, d_{X}\right)$ and $\left(Y, d_{Y}\right)$ be two metric spaces, and assume that $f: X \rightarrow Y$ is continuous. Then, a function $\phi: \mathbb{N} \rightarrow \mathbb{N}$ is said to be a modulus function for $f$ (on $X$ ) iff:

$$
\forall n \in \mathbb{N}, \forall x, y \in X: \quad d_{X}(x, y) \leq 2^{-\phi(n)} \Rightarrow d_{Y}(f(x), f(y)) \leq 2^{-n} .
$$

A fundamental property of computable real functions is that they are continuous, and over compact domains, they have a recursive modulus of continuity:

Theorem 1. Let $f:[0,1]^{n} \rightarrow \mathbb{R}$ be computable. Then, $f$ is continuous, and has a recursive modulus of continuity.

Proof. See, e. g., [15, Theorem 2.13].

\section{Distribution function and non-increasing rearrangement}

In this section, we discuss computable analysis of the distribution function $\lambda_{u}$ of a given $u:[0,1]^{n} \rightarrow \mathbb{R}$, and its non-increasing rearrangement $u^{4}$. For every $d \in \mathbb{R}$, define $A_{u}(d):=\left\{x \in[0,1]^{n} \mid u(x)>d\right\}$, and $B_{u}(d):=\left\{x \in[0,1]^{n} \mid u(x)<d\right\}$. In [15, Theorems 5.14 and 5.15], it has been shown that:

- $u$ is computable iff the classes of sets $A_{u}(d)$ and $B_{u}(d)$ (when $d$ ranges over $\mathbb{D}$ ) are uniformly recursively open.

- $u$ is recursively approximable iff the classes of sets $A_{u}(d)$ and $B_{u}(d)$ (when $d$ ranges over $\mathbb{D}$ ) are uniformly recursively $G_{\delta}$.

In both cases, by uniform, we mean uniform in $d$.

Important as they are, these results do not address the question of computability of $\lambda_{u}$. In fact, it is not difficult to see that:

Proposition 2. If $u$ has a significant flat zone, then $\lambda_{u}$ is not computable. 


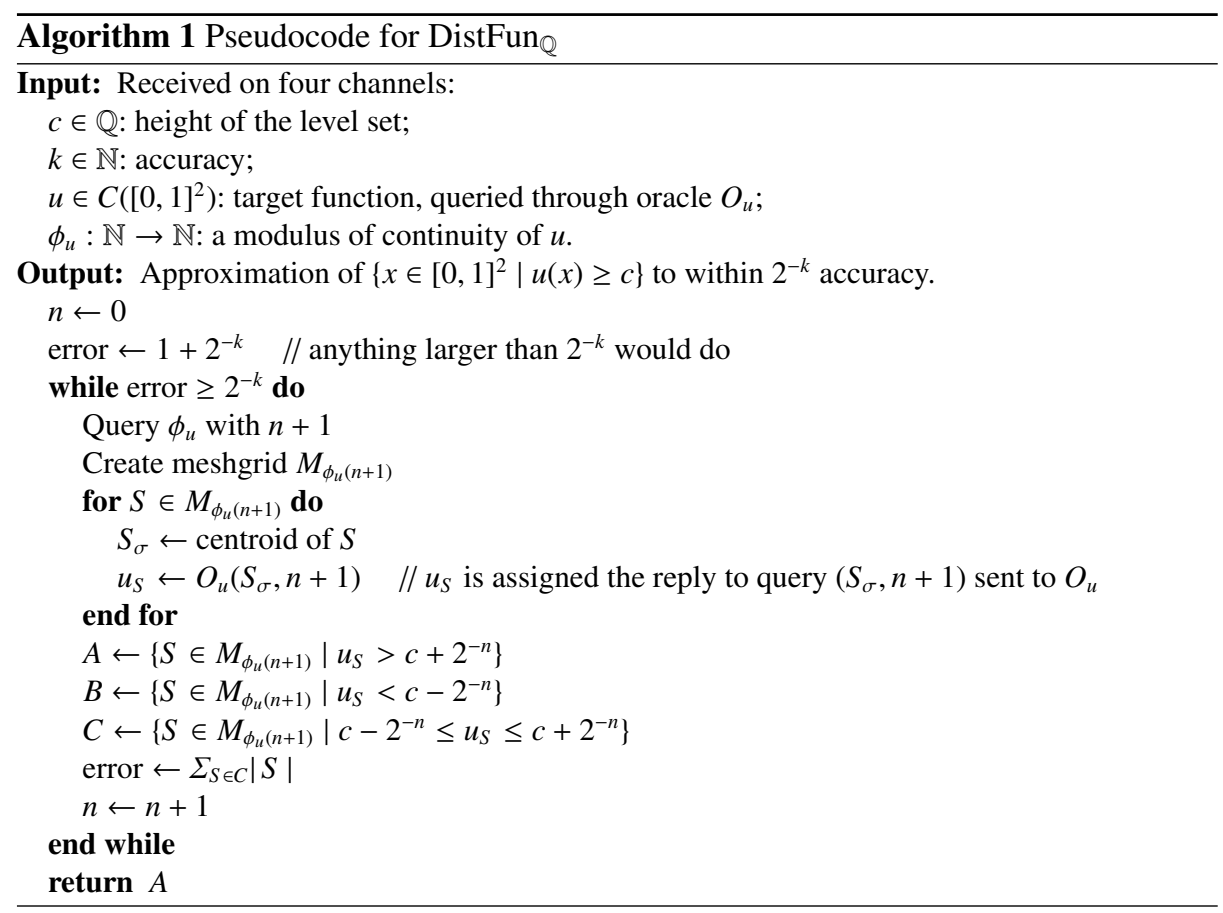

Proof. Let us assume that $u$ has a significant flat zone with value $c$. Then $\lambda_{u}$ is not continuous at $c$. By Theorem $11, \lambda_{u}$ is not computable.

Here, we consider the case where $u$ and one of its moduli of continuity are provided, respectively, as oracles $O_{u}$ and $\phi_{u}$-hence, their computability is not assumed-but we have to demand $u$ not to have any significant flat zones. For simplicity, we focus on the case $n=2$, though the results may be generalized in a straightforward way to any finite dimension.

First, we consider the machine DistFun, which operates under Algorithm 1 This machine takes a height $c \in \mathbb{Q}$ together with an accuracy parameter $k \in \mathbb{N}$, and then, through querying the oracles $O_{u}$ and $\phi_{u}$, provides an approximation of $\left\{x \in[0,1]^{2} \mid\right.$ $u(x) \geq c\}$ to within $2^{-k}$ accuracy. This can then be used to obtain a rational approximation of $\lambda_{u}(c)$ to within $2^{-k}$ accuracy. The way the oracle $O_{u}$ operates is as expected:

- On any input query $((x, y), m) \in(\mathbb{Q} \cap[0,1])^{2} \times \mathbb{N}$ received on its input channel, $O_{u}$ outputs a rational $\hat{u}:=O_{u}((x, y), m) \in \mathbb{Q}$ such that $|\hat{u}-u(x, y)| \leq 2^{-m}$.

The correctness of the algorithm for rational input values hinges on the following:

Lemma 3. Assume that $u: \Omega \rightarrow \mathbb{R}$ is continuous with no significant flat zones, where $\Omega \subseteq \mathbb{R}^{n}$ is a bounded domain. Then:

$$
\forall c \in \mathbb{R}, \forall \epsilon>0, \exists \delta>0:|\{x \in \Omega|| u(x)-c \mid<\delta\}|<\epsilon .
$$

Proof. Assume that $c$ and $\epsilon$ are given. As $u$ is continuous with no significant flat zones, the level set $u_{c}:=\{x \in \Omega \mid u(x)=c\}$ is closed and has Lebesgue measure zero. 
Hence, there exists an open set $O \subseteq \mathbb{R}^{n}$ such that $|O|<\epsilon$ and $u_{c} \subseteq O$. The function $v(x):=|u(x)-c|$ is continuous over the compact domain $\bar{\Omega} \backslash O$. Hence, it attains its minimum at some point (say) $x_{0}$, for which, we have $v\left(x_{0}\right) \neq 0$. The value $\delta:=\left|u\left(x_{0}\right)-c\right|$ satisfies (4).

Theorem 2. Assume that $u:[0,1]^{2} \rightarrow \mathbb{R}$ is a continuous function with no significant flat zones, and let $\phi_{u}: \mathbb{N} \rightarrow \mathbb{N}$ be a modulus of continuity for $u$. Then, the machine DistFun $_{\mathbb{Q}}$, operating under Algorithm 1 , halts on any input $c \in \mathbb{Q}$ and $k \in \mathbb{N}$, and returns a finite set A of two-dimensional rational boxes, such that:

- $\cup A \subseteq \alpha_{c}:=\{x \in \Omega \mid u(x) \geq c\}$, and $\left|\alpha_{c} \backslash \cup A\right| \leq 2^{-k}$.

$-q:=|\cup A| \in \mathbb{Q}$ and satisfies: $\left|q-\lambda_{u}(c)\right| \leq 2^{-k}$.

Proof. A careful inspection of Algorithm 1 reveals that, at every iteration of the while loop, we have:

$$
\cup A \subseteq \alpha_{c} \subseteq(\cup A) \cup(\cup C)
$$

To see this, assume that $S \in A$, and $S_{\sigma}$ is its centroid. For all $y \in S$, we have $\left\|y-S_{\sigma}\right\|_{\infty}<$ $2^{-\phi_{u}(n+1)}$, which, by 3 , entails that $u(y) \geq u\left(S_{\sigma}\right)-2^{-(n+1)}$. As the oracle $O_{u}$ has been queried with accuracy $n+1$, we have $u\left(S_{\sigma}\right) \geq u_{S}-2^{-(n+1)}$. Therefore $u(y) \geq u_{S}-2^{-n}>c$. This proves that $S \subseteq \alpha_{c}$, hence $\cup A \subseteq \alpha_{c}$. A similar argument shows that $\forall S \in B: \forall y \in$ $S: u(y)<c$, which proves that $\alpha_{c} \subseteq(\cup A) \cup(\cup C)$.

From (5), we obtain:

$$
|\cup A| \leq\left|\alpha_{c}\right| \leq|\cup A|+|\cup C|
$$

Now, at the $n$-th iteration of the while loop, we have:

$$
\forall x \in \cup C:|u(x)-c| \leq 2^{-n}+2^{-(n+1)}<2^{-(n-1)} .
$$

According to (4), for the given $c \in \mathbb{Q}$ and $k \in \mathbb{N}$, there exists an $n_{0} \in \mathbb{N}$ such that:

$$
\forall n \geq n_{0}:\left|\left\{x \in \Omega|| u(x)-c \mid<2^{-(n-1)}\right\}\right|<2^{-k} .
$$

From $(8)$ and (7), we infer that at iterations $n>n_{0}$, we have $|\cup C|<2^{-k}$. But $|\cup C|$ is exactly the value of error in Algorithm 1, and the output of the algorithm is $A$. This, together with (6), proves the result.

Although Theorem 2 is sufficient for our purposes, we discuss the general case where $c \in \mathbb{R}$ for completeness. As rational numbers are finitely representable, in Algorithm 1 , the value of $c$ is provided to the machine DistFun in $_{\mathbb{Q}}$ one transaction. When $c \in \mathbb{R}$, the value should be provided to the respective machine DistFun $\mathbb{R}$ (Algorithm 2) through an oracle $O_{c}$, which, on any given input $n \in \mathbb{N}$, supplies $\operatorname{DistFun}_{\mathbb{R}}$ with a rational $c_{n} \in \mathbb{Q}$ satisfying:

$$
\left|c-c_{n}\right| \leq 2^{-n}
$$

Theorem 3. Assume that $u:[0,1]^{2} \rightarrow \mathbb{R}$ is a continuous function with no significant flat zones, and let $\phi_{u}: \mathbb{N} \rightarrow \mathbb{N}$ be a modulus of continuity for $u$. Then, the machine DistFun $_{\mathbb{R}}$, operating under Algorithm 2, halts on any input $c \in \mathbb{R}$ and $k \in \mathbb{N}$, and returns a finite set $A$ of two-dimensional rational boxes, such that: 


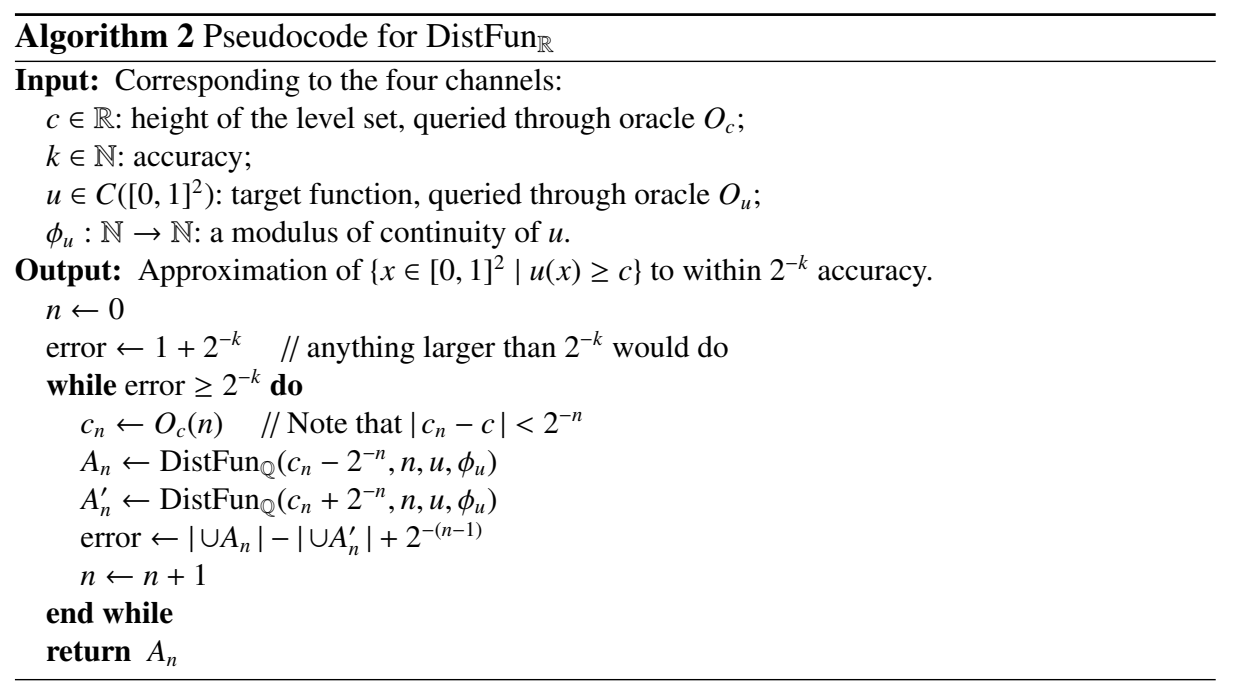

- $\cup A \subseteq \alpha_{c}:=\{x \in \Omega \mid u(x) \geq c\}$, and $\left|\alpha_{c} \backslash \cup A\right| \leq 2^{-k}$.

$-q:=|\cup A| \in \mathbb{Q}$ and satisfies: $\left|q-\lambda_{u}(c)\right| \leq 2^{-k}$.

Proof. By 9 we have $\forall n: c_{n}-2^{-n} \leq c \leq c_{n}+2^{-n}$. As $\lambda_{u}$ is decreasing (Prop. 11, we have:

$$
\lambda_{u}\left(c_{n}+2^{-n}\right)<\lambda_{u}(c)<\lambda_{u}\left(c_{n}-2^{-n}\right) .
$$

Let us define $q_{n}:=\left|\cup A_{n}\right|$ and $q_{n}^{\prime}:=\left|\cup A_{n}^{\prime}\right|$. By Theorem 2 , we have:

$$
\left\{\begin{array}{l}
\left|q_{n}-\lambda_{u}\left(c_{n}-2^{-n}\right)\right| \leq 2^{-n} \\
\left|q_{n}^{\prime}-\lambda_{u}\left(c_{n}+2^{-n}\right)\right| \leq 2^{-n}
\end{array}\right.
$$

From (10) and 111, we get:

$$
q_{n}^{\prime}-2^{-n} \leq \lambda_{u}(c) \leq q_{n}+2^{-n},
$$

which explains why error is defined as the value of $q_{n}-q_{n}^{\prime}+2^{-(n-1)}$.

Note that $\lambda_{u}$ is continuous (Prop. 11). Therefore:

$$
\lim _{n \rightarrow \infty} \lambda_{u}\left(c_{n}-2^{-n}\right)=\lim _{n \rightarrow \infty} \lambda_{u}\left(c_{n}+2^{-n}\right)=\lambda_{u}(c) .
$$

In particular $\lim _{n \rightarrow \infty} q_{n}=\lim _{n \rightarrow \infty} q_{n}^{\prime}=\lambda_{u}(c)$. This, together with (11), ensure that the aforementioned error goes below $2^{-k}$ for sufficiently large $n$. This proves halting of the algorithm.

By Prop. 1. when $u$ is continuous and has no significant flat zones, then $u^{\Delta}$ and $\lambda_{u}$ are inverses of each other. This, together with the fact that both functions are oneto-one, continuous, and decreasing, provides a simple way of obtaining the decreasing rearrangement of $u$ from its distribution function:

Corollary 1. There exists an oracle machine $\operatorname{InvDistFun}_{\mathbb{R}}$ which, given: 
- a continuous $u \in C\left([0,1]^{2}\right)$ with no significant flat zones and a modulus of continuity $\phi_{u}: \mathbb{N} \rightarrow \mathbb{N}$ for $u$

- a real number $r \in[0,1]$ and an accuracy $k \in \mathbb{N}$,

returns a rational number $c \in \mathbb{Q}$, together with a finite set of cells $A_{r} \subseteq M_{n_{0}}$, for some meshgrid of granularity $n_{0}$, such that $\left|c-u^{\Delta}(r)\right| \leq 2^{-(k+1)}, \cup A_{r} \subseteq\left\{x \in[0,1]^{2} \mid\right.$ $u(x) \geq c\}$, and $\left|\Sigma_{S \in A_{r}}\right| S|-r| \leq 2^{-(k+1)}$.

Furthermore, if $c_{r}=u^{\Delta}(r)$ and $\alpha_{c_{r}}:=\left\{x \in[0,1]^{2} \mid u(x) \geq c_{r}\right\}$, then:

$$
\left|\cup A_{r} \Delta \alpha_{c_{r}}\right| \leq 2^{-k},
$$

in which $\Delta$ denotes symmetric difference of sets defined as $X \Delta Y:=(X \backslash Y) \cup(Y \backslash X)$.

Proof. As a modulus of continuity is provided, a lower bound $a$ and an upper bound $b$ for $u$ can be easily obtained. All that remains to do for $\operatorname{InvDistFun}_{\mathbb{R}}$ is to perform a binary search using the machine DistFun $_{\mathbb{Q}}$ of Theorem 2

So far, we have only demanded the level sets of $u$ to have measure zero. By Theorem 3 , these level sets become computably measure zero provided that $u$ is computable:

Corollary 2. Assume that $u:[0,1]^{2} \rightarrow \mathbb{R}$ is a computable function with no significant flat zones. Then for any computable $c \in \mathbb{R}$, the level set $u^{-1}(c)$ is computably measure zero.

\section{Linear rearrangement optimization}

Assume that, for some $p \in[1, \infty)$, we are given a generator $f_{0} \in L^{p}([0,1])$ and a function $u \in C\left([0,1]^{2}\right)$ which has no significant flat zones. Our task is to compute the necessarily unique $\hat{f} \in \mathcal{R}_{[0,1]^{2}}\left(f_{0}\right)$ which maximizes the functional $L_{u}$ as defined in 11 . Equation (2) provides the basis for the results of this section. Nonetheless, as $f_{0}$ is in $L^{p}([0,1])$ — hence might have discontinuities —we need to go through some careful computable analysis to make sure that error estimates are accurately accounted for.

Requiring $u$ to be continuous might seem like a strong condition, but in practice, solutions of the PDE constraints to rearrangement optimization problems that we have in mind invariably are 'continuous', i. e., the solutions lie in $C(\bar{\Omega})$, where $\Omega$ is the domain over which the PDE is stated. Furthermore, requiring $u$ not to have any significant flat zones ensures uniqueness of solutions, and again, it is a condition that is satisfied in the vast majority of PDE-constrained rearrangement optimization problems in the literature.

For computational purposes, the function $u$ and one of its moduli of continuity $\phi_{u}$ will be provided as oracles, in the same manner as in Sect. 3. As for the generator $f_{0}$, we first note that for any given $p \in[1, \infty)$, the set of simple step functions is dense in $L^{p}([0,1])$. Thus, we represent $f_{0} \in L^{p}([0,1])$ as the limit of a Cauchy sequence of simple step functions converging to it. Using the results of Sect. 3 , we obtain tight approximations of linear rearrangement maximizers for the approximant simple step functions, and then prove that these approximations, in turn, converge to the true maximizer for the given (potentially not finitely representable) function $f_{0}$. 
Remark 2. Although we focus on maximization, corresponding results for linear rearrangement minimization may be obtained with straightforward tweaking of the arguments and the proofs.

\subsection{Simple step function generator}

For some $p \in[1, \infty)$, assume that $f \in L^{p}([0,1])$ is a simple step function, represented by the set:

$$
\left\{x_{0}, x_{1}, \ldots, x_{n}, y_{1}, \ldots, y_{n}\right\} \subseteq \mathbb{Q},
$$

Without loss of generality, we assume that $f$ is non-increasing and $\forall i \neq j: y_{i} \neq y_{j}$. If this is not the case, a simple sorting and then gluing of subintervals can ensure these two conditions. Let $\hat{f}$ be the unique maximizer of $L_{u}$ over $\mathcal{R}_{[0,1]^{2}}(f)$ as in Lemma 1 , vid, and let $\gamma:=2 \max \left\{\left|y_{i}\right| \mid 1 \leq i \leq n\right\}$.

Now, assume that we are given an $\epsilon>0$, and our aim is to find some $\tilde{f}$ which approximates $\hat{f}$ to within $\epsilon$ accuracy. Let $k \in \mathbb{N}$ be large enough such that:

$$
2^{-k}<\frac{\epsilon^{p}}{n \gamma^{p}}
$$

Together with $u$, we provide this value $k$, and successive values of $x_{i}$, to the machine InvDistFun $_{\mathbb{R}}$, and for each $i \in\{1, \ldots, n\}$, let $A_{x_{i}}$ be as in Corollary 1 . In particular, if $c_{i}:=\lambda_{u}^{-1}\left(x_{i}\right)$ and $\alpha_{c_{i}}:=\left\{x \in[0,1]^{2} \mid u(x) \geq c_{i}\right\}$, then by 12 we know that $\cup A_{i}$ approximates $\alpha_{c_{i}}$ to within $2^{-k}$ accuracy. Next, we define:

$$
\left\{\begin{array}{l}
\hat{A}_{x_{1}}:=\cup A_{x_{1}}, \\
\hat{A}_{x_{k}}:=\cup A_{x_{k}} \backslash \cup A_{x_{k-1}}, \quad(2 \leq k \leq n),
\end{array}\right.
$$

and note that $\hat{A}_{x_{i}}$ 's partition $[0,1]^{2}$. Hence, we can define a piecewise constant function $\tilde{f}:[0,1]^{2} \rightarrow \mathbb{R}$ as follows:

$$
\forall x \in[0,1]^{2}: \tilde{f}(x):=\sum_{i=1}^{n} y_{i} \chi_{\hat{A}_{x_{i}}}(x) .
$$

From Corollary 1 , we deduce that $\tilde{f}$ and $\hat{f}$ coincide on all of $[0,1]^{2}$ except perhaps on a set of Lebesgue measure at most $n 2^{-k}$. Thus:

$$
\begin{aligned}
\left(\int_{[0,1]^{2}}|\tilde{f}(x)-\hat{f}(x)|^{p} \mathrm{~d} x\right)^{1 / p} & <\left(n 2^{-k} \gamma^{p}\right)^{1 / p} \\
(\text { by }(13)) & <\epsilon .
\end{aligned}
$$

Putting all of the above together, we obtain:

Lemma 4. There exists an oracle machine $M_{1}$ which, given:

- a continuous $u \in C\left([0,1]^{2}\right)$ with no significant flat zones, and a modulus of continuity $\phi_{u}: \mathbb{N} \rightarrow \mathbb{N}$ for $u$;

- a real number $p \in[1, \infty)$, and a simple step function $f \in L^{p}([0,1])$;

- an accuracy parameter $n \in \mathbb{N}$;

returns a piecewise constant function $\tilde{f} \in L^{p}\left([0,1]^{2}\right)$ such that $\|\tilde{f}-\hat{f}\|_{p}<2^{-n}$, in which $\hat{f}$ is the unique maximizer of $L_{u}$ over $\mathcal{R}_{[0,1]^{2}}(f)$ as in Lemma 1 (vi). 
Fig. 1 An oracle machine representation of LinMax, which computes the linear rearrangement maximizer $\hat{f}$ of $L_{u}$ over $\mathcal{R}_{[0,1]^{2}}(f)$.

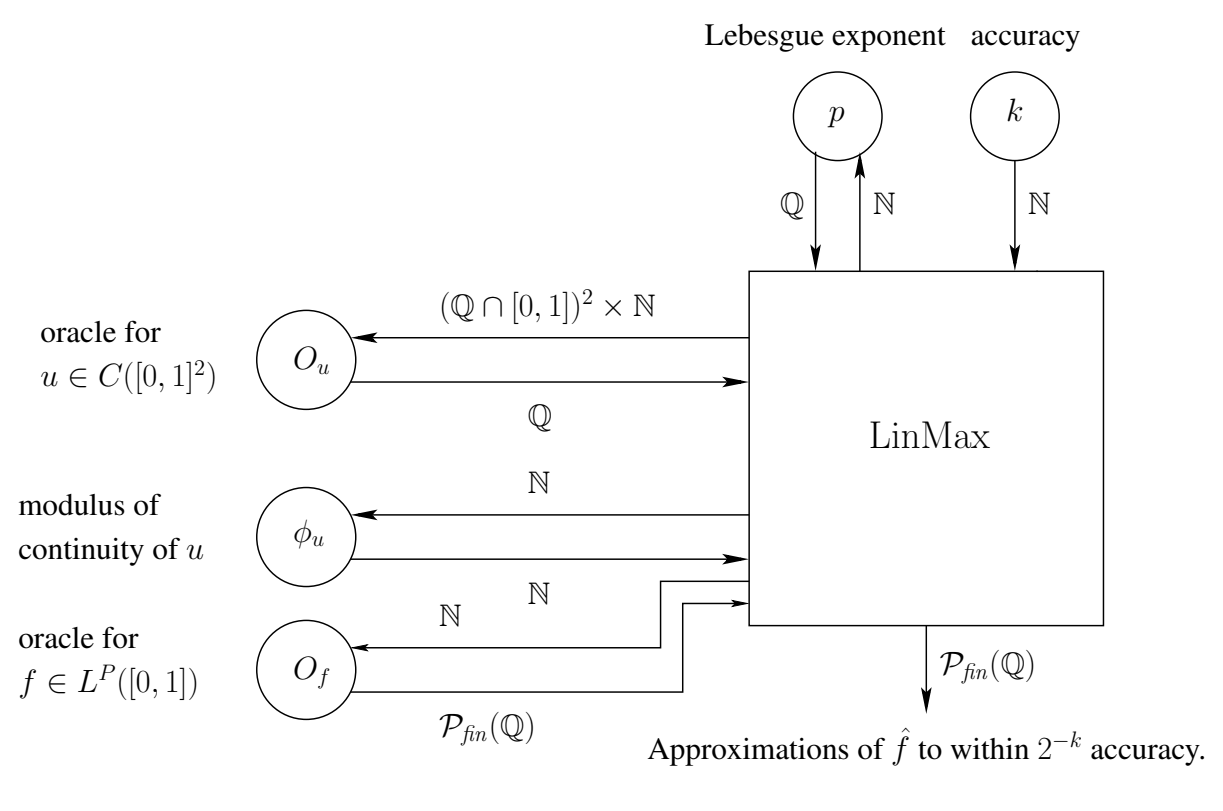

\subsection{General case}

For an arbitrary $f \in L^{P}([0,1])$, we consider the oracle machine LinMax of Fig. 1] On receiving the accuracy demand $k \in \mathbb{N}$, the machine sends $k+1$ as a query to the oracle $O_{f}$, which in turn returns a simple step function $f_{k+1}:[0,1] \rightarrow \mathbb{R}$ satisfying:

$$
\left\|f_{k+1}-f\right\|_{p} \leq 2^{-(k+1)} .
$$

Subsequently, LinMax uses the machine $M_{1}$ from Lemma 4 providing it with $p, u$, $\phi_{u}, f_{k+1}$, and accuracy parameter $k+1$. The machine $M_{1}$, in turn, returns a piecewise constant $\tilde{f}_{k+1} \in L^{P}\left([0,1]^{2}\right)$ which satisfies:

$$
\left\|\tilde{f}_{k+1}-\hat{f}_{k+1}\right\|_{p} \leq 2^{-(k+1)} .
$$

Finally, LinMax returns $\tilde{f}_{k+1}$ as output.

To prove that LinMax is working correctly, we need to prove that:

$$
\left\|\tilde{f}_{k+1}-\hat{f}\right\|_{p} \leq 2^{-k} \text {. }
$$

By Lemma 2 (i), there exists a measure-preserving map $\rho:[0,1]^{2} \rightarrow[0,1]$ satisfying:

$$
u=u^{4} \circ \rho,
$$

where $u^{\Delta}$ is the decreasing rearrangement of $u$. As $\hat{f}$ and $\hat{f}_{k+1}$ are maximizers of $L_{u}$ over $\mathcal{R}_{[0,1]^{2}}(f)$ and $\mathcal{R}_{[0,1]^{2}}\left(f_{k+1}\right)$, respectively, by Lemma 1 |vi], there are non-decreasing 
functions $\psi_{1}$ and $\psi_{2}$ such that:

$$
\hat{f}=\psi_{1} \circ u \quad \text { and } \quad \hat{f}_{k+1}=\psi_{2} \circ u .
$$

As $\psi_{1}$ and $\psi_{2}$ are non-decreasing, it is straightforward to show that:

$$
f^{\Delta}=\psi_{1} \circ u^{\Delta} \quad \text { and } \quad f_{k+1}^{\Delta}=\psi_{2} \circ u^{\Lambda} .
$$

From $17,, 18$, and 19 , we deduce $\hat{f}=f^{\Delta} \circ \rho$ and $\hat{f}_{k+1}=f_{k+1}^{\Delta} \circ \rho$, which implies that:

$$
\begin{aligned}
\left\|\hat{f}-\hat{f}_{k+1}\right\|_{p} & =\left\|f^{\Delta}-f_{k+1}^{\Delta}\right\|_{p} \\
(\text { by Lemma } 2 \text { (1ii) }) & \leq\left\|f_{k+1}-f\right\|_{p} \\
(\text { by } 140) & \leq 2^{-(k+1)} .
\end{aligned}
$$

By combining 15 and 20 , we obtain 16 . Hence, we have:

Theorem 4. There exists an oracle machine LinMax which, given:

- a continuous $u \in C\left([0,1]^{2}\right)$ with no significant flat zones, and a modulus of continuity $\phi_{u}: \mathbb{N} \rightarrow \mathbb{N}$ for $u$;

- a real number $p \in[1, \infty)$, and a function $f \in L^{p}([0,1])$;

- an accuracy parameter $k \in \mathbb{N}$;

returns a piecewise constant function $\tilde{f} \in L^{p}\left([0,1]^{2}\right)$ such that $\|\tilde{f}-\hat{f}\|_{p} \leq 2^{-k}$, in which $\hat{f}$ is the unique maximizer of $L_{u}$ over $\mathcal{R}_{[0,1]^{2}}(f)$ as in Lemma 1 (vi).

Essentially, we have fleshed out the algorithm for computing $\hat{f}$ which is suggested by equation (2). If we define $\psi:=f^{\Delta} \circ \lambda_{u}$, then we will have $\hat{f}=\psi \circ u$. Now it should be clear that the measure-preserving transformation $\rho$ of (17) is just $\lambda_{u} \circ u$. Note that $\lambda_{u}$ can be obtained by Theorem 3 , and $f^{\Delta}$ may be approximated using the simple step function approximations $\left\{f_{n} \mid n \in \mathbb{N}\right\}$ of $f$.

\section{Generalizations}

To stay focused on the essence of rearrangements, we presented our results for the simple two dimensional cube $[0,1] \times[0,1]$. Generalizations to the following, however, are straightforward:

- Linear rearrangement minimization;

- $n$-dimensional cube $[0,1]^{n}$, for all $n \in \mathbb{N}$;

- Open, bounded, and connected domains $\Omega \subseteq \mathbb{R}^{n}$, for which $\bar{\Omega}$ is a union of $n$ dimensional cubes with rational coordinates.

Some careful error analysis, together with (say) Delaunay triangulation, may provide a further generalization to polygonal domains with rational coordinates. Indeed, domains $\Omega$ that can be approximated from within via rational polygonal domains, whose 
boundary vertices lie on the boundary of $\Omega$, may also be treated, using more careful error analysis.

Going further to general domains might need substantial change in approach, especially if the approximants of the domain $\Omega$ have to cover locations out of $\Omega$. This is reminiscent of the finite element methods for numerical solutions of PDEs, where care is taken to have the finite element space as a subspace of the reference Sobolev space.

\section{Conclusions and future work}

We have taken some steps towards computable analysis of rearrangement optimization problems. We provided oracle Turing machines that compute the distribution function, decreasing rearrangement, and linear rearrangement optimizers, with respect to functions that are continuous and have no significant flat zones.

The next step will be the computable analysis of a complete PDE-constrained rearrangement optimization problem. Note that linear rearrangement optimization is one of the two main components of some numerical methods for solving PDE-constrained rearrangement optimization problems [7]10], the other being PDE solving. Apart from some isolated work (e.g., [2]16]) computable analysis of PDE solving is largely an unexplored area.

In longer term, we aim to develop validated methods for shape optimization and free boundary problems arising as PDE-constrained rearrangement optimization problems.

\section{References}

1. Benjamin, T.B.: The alliance of practical and analytical insights into the nonlinear problems of fluid mechanics. In: Lecture Notes in Mathematics, vol. 503, pp. 8-29. Springer (1976)

2. Brattka, V., Yoshikawa, A.: Towards computability of elliptic boundary value problems in variational formulation. Journal of Complexity 22(6), 858-880 (2006)

3. Burton, G.R.: Rearrangements of functions, maximization of convex functionals, and vortex rings. Math. Ann. 276(2), 225-253 (1987)

4. Burton, G.R.: Variational problems on classes of rearrangements and multiple configurations for steady vortices. Ann. Inst. H. Poincaré Anal. Non Linéaire 6(4), 295-319 (1989)

5. Burton, G.R., McLeod, J.B.: Maximisation and minimisation on classes of rearrangements. Proc. Roy. Soc. Edinburgh Sect. A 119(3-4), 287-300 (1991)

6. Crowe, J.A., Zweibel, J.A., Rosenbloom, P.C.: Rearrangements of functions. J. Funct. Anal. 66(3), 432-438 (1986)

7. Elcrat, A., Nicolio, O.: An iteration for steady vortices in rearrangement classes. Nonlinear Anal. 24(3), 419-432 (1995)

8. Emamizadeh, B., Marras, M.: Rearrangement optimization problems with free boundary. Numer. Funct. Anal. Optim. 35(4), 404-422 (2014)

9. Emamizadeh, B., Zivari-Rezapour, M.: Rearrangements and minimization of the principal eigenvalue of a nonlinear Steklov problem. Nonlinear Anal. 74(16), 5697-5704 (2011)

10. Emamizadeh, B., Farjudian, A., Liu, Y.: Optimal harvesting strategy based on rearrangements of functions. Applied Mathematics and Computation 320, 677-690 (2018)

11. Emamizadeh, B., Farjudian, A., Zivari-Rezapour, M.: Optimization related to some nonlocal problems of Kirchhoff type. Canad. J. Math. 68(3), 521-540 (2016) 
12. Emamizadeh, B., Hanai, M.A.: Rearrangements in real estate investments. Numerical Functional Analysis and Optimization 30(5-6), 478-485 (2009)

13. Farjudian, A.: Computable analysis of linear rearrangement optimization. In: Gopal, T.V., Watada, J. (eds.) Theory and Applications of Models of Computation (TAMC 2019). Lecture Notes in Computer Science, vol. 11436, pp. 172-187. Springer (2019). https://doi.org/10.1007/978-3-030-14812-6_11

14. Kao, C.Y., Su, S.: Efficient rearrangement algorithms for shape optimization on elliptic eigenvalue problems. J. Sci. Comput. 54(2), 492-512 (2013)

15. Ko, K.I.: Complexity Theory of Real Functions. Birkhäuser, Boston (1991)

16. Selivanova, S., Selivanov, V.: Computing the solution operators of symmetric hyperbolic systems of PDE. Journal of Universal Computer Science 15(6), 1337-1364 (2009)

17. Talenti, G.: The art of rearranging. Milan J. Math. 84(1), 105-157 (2016)

18. Weihrauch, K.: Computable Analysis, An Introduction. Springer (2000) 